Delft University of Technology

\title{
Combining diffuse reflectance spectroscopy and ultrasound imaging for resection margin assessment during colorectal cancer surgery
}

Geldof, Freija; Jong, Lynn-Jade; Dashtbozorg, Behdad; Hendriks, Bernardus H.W.; Sterenborg, Henricus J.C.M.; Ruers, Theo J.M.

DOI

10.1117/12.2578478

Publication date

2021

Document Version

Final published version

Published in

Multimodal Biomedical Imaging XVI

\section{Citation (APA)}

Geldof, F., Jong, L-J., Dashtbozorg, B., Hendriks, B. H. W., Sterenborg, H. J. C. M., \& Ruers, T. J. M.

(2021). Combining diffuse reflectance spectroscopy and ultrasound imaging for resection margin assessment during colorectal cancer surgery. In F. S. Azar, X. Intes, \& Q. Fang (Eds.), Multimodal Biomedical Imaging XVI [116340K] (Progress in Biomedical Optics and Imaging - Proceedings of SPIE; Vol. 11634). SPIE. https://doi.org/10.1117/12.2578478

Important note

To cite this publication, please use the final published version (if applicable).

Please check the document version above. 


\section{Combining diffuse reflectance spectroscopy and ultrasound imaging for resection margin assessment during colorectal cancer surgery}

Geldof, Freija, Jong, Lynn-Jade, Dashtbozorg, Behdad, Hendriks, Bernardus H., Sterenborg, Henricus J. C., et al.

Freija Geldof, Lynn-Jade Jong, Behdad Dashtbozorg, Bernardus H. W. Hendriks, Henricus J. C. M. Sterenborg, Theo J. M. Ruers, "Combining diffuse reflectance spectroscopy and ultrasound imaging for resection margin assessment during colorectal cancer surgery," Proc. SPIE 11634, Multimodal Biomedical Imaging XVI, 116340K (9 March 2021); doi: 10.1117/12.2578478 


\title{
Combining diffuse reflectance spectroscopy and ultrasound imaging for resection margin assessment during colorectal cancer surgery
}

\author{
Freija Geldof ${ }^{* a}$, Behdad Dashtbozorg ${ }^{\mathrm{a}}$, Benno H.W. Hendriks ${ }^{\mathrm{b}, \mathrm{c}}$, Henricus J.C.M. Sterenborg ${ }^{\mathrm{a}, \mathrm{d}}$, Theo \\ J.M. Ruers ${ }^{\mathrm{a}, \mathrm{e}}$ \\ a Department of Surgery, Netherlands Cancer Institute, Plesmanlaan 121, 1066CX Amsterdam, \\ Netherlands; ${ }^{b}$ Department of In-body Systems, Philips Research, Eindhoven, 5656 AE, The \\ Netherlands; ${ }^{\mathrm{c}}$ Department of Biomedical Engineering, Delft University of Technology, Mekelweg 2 \\ 2628 CD, Delft, The Netherlands; ${ }^{\mathrm{d}}$ Department of Biomedical Engineering and Physics, Amsterdam \\ University Medical Center, Meibergdreef 9, 1105 AZ Amsterdam, The Netherlands; ${ }^{\text {e }}$ Faculty of \\ Science and Technology, University of Twente, Drienerlolaan 5, 7522 NB Enschede, The \\ Netherlands \\ *f.geldof@nki.nl
}

\begin{abstract}
Establishing adequate resection margins during colorectal cancer surgery is challenging. Currently, in up to $30 \%$ of the cases the tumor is not completely removed, which emphasizes the lack of a real-time tissue discrimination tool that can assess resection margins up to multiple millimeters in depth. Therefore, we propose to combine spectral data from diffuse reflectance spectroscopy (DRS) with spatial information from ultrasound (US) imaging to evaluate multi-layered tissue structures. First, measurements with animal tissue were performed to evaluate the feasibility of the concept. The phantoms consisted of muscle and fat layers, with a varying top layer thickness of 0-10 mm. DRS spectra of 250 locations were obtained and corresponding US images were acquired. DRS features were extracted using the wavelet transform. US features were extracted based on the graph theory and first-order gradient. Using a regression analysis and combined DRS and US features, the top layer thickness was estimated with an error of up to $0.48 \mathrm{~mm}$. The tissue types of the first and second layers were classified with accuracies of 0.95 and 0.99 respectively, using a support vector machine model.
\end{abstract}

Keywords: Multimodal imaging, diffuse reflectance spectroscopy, ultrasound imaging, multi-layer tissue, surgical guidance, margin assessment, tissue classification, colorectal cancer

\section{INTRODUCTION}

In oncological surgery, the aim is to remove the entire tumor together with a margin of healthy tissue, while other surrounding healthy structures are spared as much as possible. However, identifying the tumor during surgery can be challenging. Inadequate tumor resection results in increased risks of local tumor recurrence and decreased survival rates, making the resection margin status an important prognostic factor for patients [1]. This emphasizes the demand for a realtime tissue discrimination technique to provide intra-operative guidance.

In recent years, techniques based on diffuse reflectance spectroscopy (DRS) demonstrated great potential for real-time tissue assessment during surgery [2]-[5]. In diffuse reflectance spectroscopy (DRS), broadband light in the visual and/or near-infrared range is sent into tissue using optical fibers. The detected diffuse reflectance contains information about the tissue composition. However, two main limitations arise regarding the evaluation of complete resection margins up to 5 $\mathrm{mm}$ in depth. Firstly, it is essential to distinguish different tissue layers and detect tumor even when it is below a layer of healthy tissue. Secondly, currently used analytical models for DRS spectra analysis are expected to fail for more complex multi-layered situations that will be encountered in clinical practice. To solve these problems, information about the tissue architecture is needed in addition to the spectral data. Ultrasound (US) imaging can provide images of this tissue architecture with a resolution smaller than $1 \mathrm{~mm}$ and more than sufficient sampling depth. However, US imaging alone is not representative enough for precise tissue classification. Therefore, given the strengths and weaknesses of both

Multimodal Biomedical Imaging XVI, edited by Fred S. Azar, Xavier Intes, Qianqian Fang, Proc. of SPIE Vol. 11634, 116340K · @ 2021 SPIE · CCC code: 1605-7422/21/\$21 · doi: 10.1117/12.2578478 
techniques, we propose to combine spectral data of DRS with spatial information of US to obtain features that surpass the capacity of each technique separately. This enables analysis of more complex structures and extraction of information from larger sampling depths.

The aim of this study is to develop algorithms in which spectral information and ultrasound information will be combined for the assessment of multi-layered tissue structures. The performance of the algorithms in predicting the top layer thickness and classifying the tissue types of the layers will be examined on animal tissue as a proof of concept.

\section{MATERIALS AND METHODS}

\subsection{Data acquisition and preprocessing}

Eight pieces of bovine and porcine meat were used in this study. The animal tissue phantoms consisted of two different homogeneous layers; a bottom layer of at least $20 \mathrm{~mm}$ thick and a top layer with a thickness varying between 0 and 10 $\mathrm{mm}$. Two types of phantoms were examined; muscle tissue with a top layer of fat and fat with a top layer of muscle tissue. The measurements were performed in a grid-pattern. At every location, first a DRS measurement was performed. The DRS system consisted of a Tungsten halogen broadband light source and two spectrometers; one for the visible range of 400 to $1100 \mathrm{~nm}$ (Andor Technology, DU420ABRDD) and one for the near-infrared range of 800 to $1700 \mathrm{~nm}$ (Andor Technology, DU492A-1.7) [6]. Source-detector fiber distances of 2 and $6 \mathrm{~mm}$ were used to derive information about the tissue composition from different sampling depths. Subsequently, an US image was acquired in such a way that the location of the DRS measurement corresponded to the center of the US image. Two US devices have been used (Philips, CX50 and Philips, EPIQ 7) with two different transducers (Philips, L15-7io and Philips, eL18-4 respectively), to avoid dependence on a particular system.

Measurements were performed at 250 locations in total; 122 fat on top of muscle and 128 muscle on top of fat measurements. For each spectrum, the corresponding top layer thickness was determined based on the US image (ground truth in this study). Only the measurement locations associated with a top layer thickness smaller than $6 \mathrm{~mm}(\mathrm{n}=186)$ were included for further analyses, since it is expected that the sampling depth of DRS will be equal to the fiber distance of $6 \mathrm{~mm}$ and there is also no clinical interest to assess tissue deeper than $6 \mathrm{~mm}$. To compensate for any intensity differences due to differences in for instance pressure applied to the tissue, the spectra were normalized with respect to the intensity value at $800 \mathrm{~nm}$.

\subsection{Spectral feature extraction}

Two types of features were extracted from the DRS spectra; features based on spectral peaks and features based on the wavelet transform. For the peak based method, the dip-peak height was calculated for three spectral regions with distinct peaks: $935 \mathrm{~nm}$ (fat absorption peak), $985 \mathrm{~nm}$ (water absorption peak) and $1200 \mathrm{~nm}$ (fat absorption peak), see Figure 1. These peak heights were calculated by subtracting the minimum intensity value from the maximum intensity value in the regions $920-960 \mathrm{~nm}, 960-1150 \mathrm{~nm}$ and 1150-1325 nm, respectively. Extracting the three peak heights for both fiber distances resulted in six features.

The second feature extraction method was based on the wavelet transform, which can be used to analyze spectra at different spectral scales. For this study, the dyadic implementation of the transform, as described by Denstedt et al., was used [7]. The input spectra were convolved with a Symlet low-pass filter of size 8 to obtain approximation spectra and with a Symlet high-pass filter of size 8 to obtain details spectra. Subsequently, the obtained spectra were down-sampled by a factor of 2 and the procedure was repeated multiple times, each time using the approximation spectrum of the previous iteration. The approximation spectra of the 6th iteration, consisting of 25 features, were used for the analyses. Extracting these features for both fiber distances resulted in 50 features. 

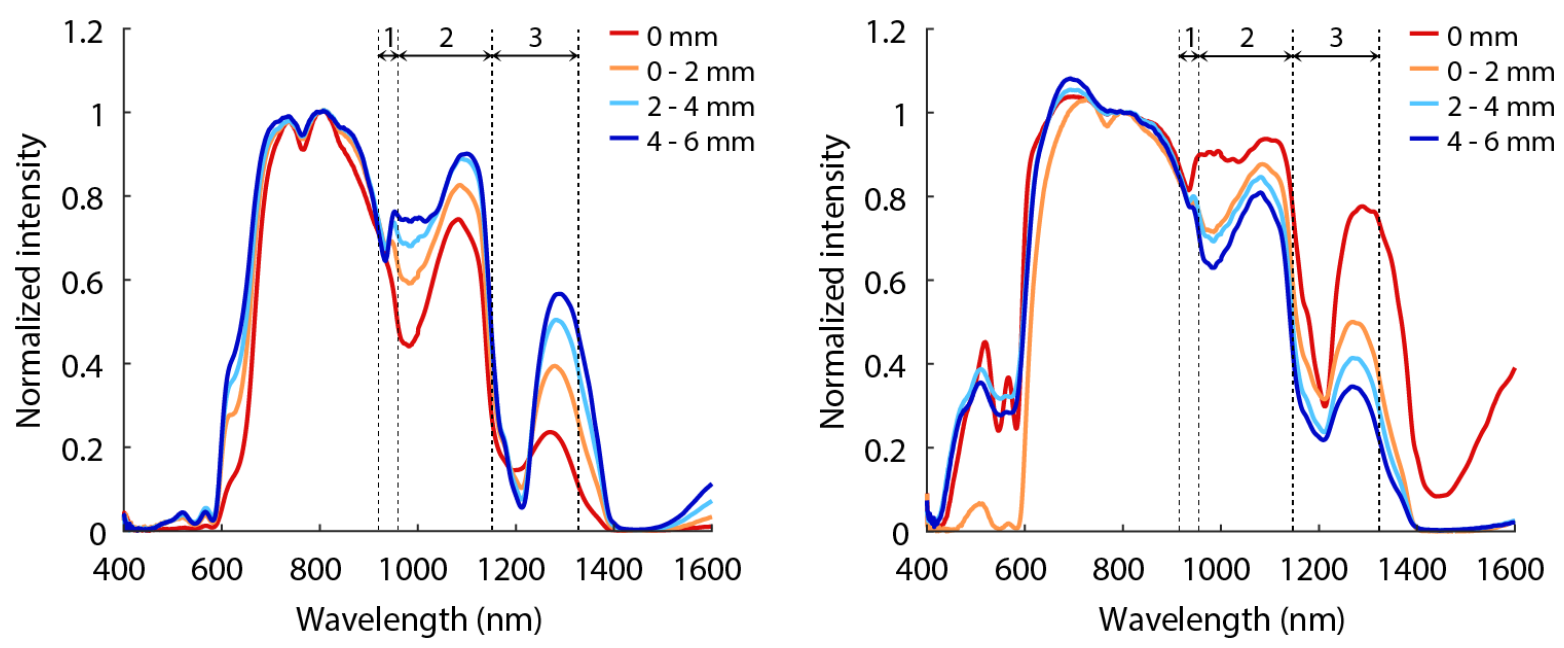

Figure 1. Mean diffuse reflectance spectra corresponding to different top layer thicknesses of fat on top of muscle (left image) and muscle on top of fat (right image), for a fiber distance of $6 \mathrm{~mm}$. The peak height was determined for the three outlined regions.

\subsection{Ultrasound feature extraction}

The US images were analyzed based on the graph theory, in which an image is represented as a graph with nodes and edges, representing the individual pixels and the connections between two neighboring pixels, respectively. The specific method used in this study was based on an automatic segmentation algorithm provided by Chiu et al. [8]. Although the algorithm was designed for the segmentation of retinal layers, it is suitable for layered structures in general and some modifications were made in order to find the fat to muscle and muscle to fat boundaries. First, the image was smoothed using a Gaussian filter $(5 \times 5 ; \sigma=1)$ and the vertical gradient image was obtained. Subsequently, a weight was assigned to each of the edges in such a way that the edges of pixel pairs with the highest vertical gradients have the lowest weights. Boundaries were selected based on minimal cost, for which Dijkstra's algorithm was used [9]. The boundary corresponding to the contact surface between the transducer and the phantom was selected using the first twenty pixel rows only. Beneath this boundary, the three best paths were selected since the first best path does not always correspond to the correct boundary. Figure 2 shows an example of the three selected paths, within this case the second path found being the correct boundary we are looking for.

The selected boundaries were translated into six features that can be used in the estimation of the top layer thickness. For each of the three selected boundaries, the distance from the surface and the vertical gradient value at the location of the path were used as feature values.

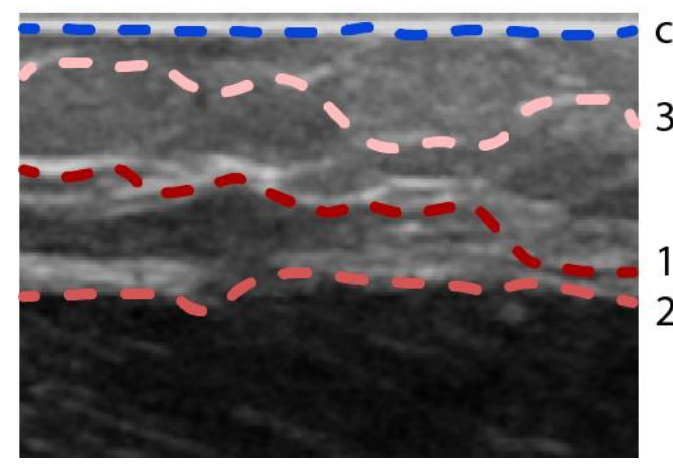

\section{contact surface}

3

Figure 2. US image of a fat layer on top of muscle measurement. The blue line is the contact surface as selected by the graph theory and the red lines show the first, second and third path best paths that were selected. 


\subsection{Top layer thickness prediction and tissue classification}

For the top layer thickness estimation, the data set was divided into two groups. One group consisted of the muscle measurements with and without a top layer of fat and one group consisted of the fat measurements with and without a top layer of muscle. For each group separately, the thickness estimation analysis was performed using a linear support vector machine (SVM) regression model [10]. For the peaks method, the analyses were based on 14 features (6 DRS and 6 US features) and for the wavelet method, the analyses were based on 56 features (50 DRS and 6 US features).

The tissue types of both layers were classified independently of each other using quadratic SVM classification models, based on the DRS features only (6 and 50 features for the peaks method and the wavelet method, respectively). For both the top layer thickness estimation and the classification, 10-fold cross-validation was performed.

\section{RESULTS}

\subsection{Top layer thickness}

After performing a regression analysis using both feature extraction methods, in the case of fat on top of the muscle tissue mean top layer thickness prediction errors of $0.75 \pm 0.67 \mathrm{~mm}$ and $0.72 \pm 0.69 \mathrm{~mm}$ were found for the peak-based method and the wavelet transform based method, respectively. In the case of muscle on top of fat tissue, mean prediction errors of $0.60 \pm 0.93 \mathrm{~mm}$ and $0.48 \pm 0.39 \mathrm{~mm}$ were achieved for the peak based method and the wavelet transform based method, respectively (see Table 1).

Table 1: Top layer thickness prediction errors, for both tissue types and feature extraction methods.

\begin{tabular}{lcc}
\hline Features & Fat on muscle & Muscle on fat \\
\hline DRS peak heights + US & $0.75 \pm 0.67 \mathrm{~mm}$ & $0.60 \pm 0.93 \mathrm{~mm}$ \\
DRS wavelet transform + US & $0.72 \pm 0.69 \mathrm{~mm}$ & $0.48 \pm 0.39 \mathrm{~mm}$ \\
\hline
\end{tabular}

\subsection{Tissue classification}

All 186 measurements were used for tissue classification of the first layer; 89 with a top layer of fat and 97 with a top layer of muscle. Tissue classification of the second layer was performed using only the two-layer measurements (73 with a second layer of fat and 81 with a second layer of muscle). Table 2 shows the results of both layers for the peak heights method and for the wavelet method. The classification accuracies for respectively the first layer and the second layer were 0.95 and 0.95 using the peak heights method and 0.95 and 0.99 using the wavelet method.

Table 2. Tissue classification scores for both the first and second layers, using the two different feature extraction methods.

\begin{tabular}{lcccc}
\hline & \multicolumn{2}{c}{ First tissue layer } & \multicolumn{2}{c}{ Second tissue layer } \\
\cline { 2 - 5 } & Peak heights & Wavelet transform & Peak heights & Wavelet transform \\
\hline Accuracy & 0.95 & 0.95 & 0.95 & 0.99 \\
Sensitivity* & 0.92 & 0.94 & 0.98 & 0.98 \\
Specificity** & 0.97 & 0.96 & 0.92 & 1.00 \\
\hline *Correctly classified fat layers, **Correctly classified muscle layers &
\end{tabular}




\section{DISCUSSION}

In this study, information from spectral and ultrasound data was combined to assess multi-layered tissue structures. Figure 1 shows a clear correlation between the top layer thickness and the spectral peak height. This can be explained by the fact that the smaller the top layer thickness, a larger part of the light travels through the second layer and the tissue type of the second layer becomes more visible in the DRS spectrum.

By performing a regression analysis, the thickness of the first layer could be predicted with an inaccuracy of less than 1 $\mathrm{mm}$. These results are promising since the errors are in the same order of magnitude as the US resolution and smaller than the resection margin of a few millimeters that is generally used during oncological surgery. The prediction error for the animal tissue phantoms with a top layer of muscle is smaller than for a top layer of fat. The results of the boundary selection in the US images might play a role in this difference. In $5.2 \%$ of the images, the correct boundary was not selected, which were all fat on top of muscle images. This might be because the boundary between the first and second layer was more clear on the US images with a top layer of muscle. When comparing the two different feature extraction methods used in this study, the mean prediction errors using the wavelet-based method $(0.72 \mathrm{~mm}$ and $0.48 \mathrm{~mm})$ are slightly smaller than using the peak based method $(0.75 \mathrm{~mm}$ and $0.60 \mathrm{~mm})$. However, the question is whether this difference will be clinically relevant in the end.

Previous studies in literature focused primarily on identifying tissue directly at the surface. In this study, good classification accuracies were achieved for both the first and second layers by combining the information about the tissue composition and architecture. The tissue type of the first layer was identified with an accuracy of 0.95 for both feature extraction methods, while the wavelet transform method performed better for the second layer with an accuracy of 0.99 . The most striking difference lies in the specificity of $100 \%$ for the wavelet transform method, which positively influenced the accuracy. The main difference between the two feature extraction methods is that the peak-based method focuses on certain regions in the spectra, while the wavelet transform uses the entire spectrum. This may explain why the wavelet transform method performs better in some situations.

In this study, two types of animal tissue phantoms were used to examine the algorithm performance. However, it still concerned a simple set-up to test the feasibility of the concept. In clinical practice, more tissue types can be encountered. Ultimately, the challenge will be to distinguish tumor tissue from multiple types of healthy tissue. A next step would therefore be to test the performance of the algorithms in more complex situations with human tissue, as in an ex vivo study.

\section{CONCLUSION}

The aim of this study was to evaluate multi-layered animal tissue by combining diffuse reflectance spectroscopy and ultrasound imaging. It was shown that the top layer thickness could be predicted with an inaccuracy of less than $1 \mathrm{~mm}$ and the first and second layer tissue type could be classified with an accuracy of 0.95 and 0.99 , respectively. These results show the potential ability of the proposed technique in revealing more complex tissue architectures. Therefore, further ex vivo studies will be carried out, keeping in mind the ultimate goal of evaluating resection margins up to multiple millimeters in depth during oncological surgery.

\section{ACKNOWLEDGMENTS}

This work was supported by KFW-STW (15022) and Philips Research, Eindhoven, The Netherlands. We are grateful for their support.

BHWH is affiliated with Philips Research; he is an employee, however, he has no financial interest in the subject matter, materials and/or equipment. None of the other authors have relevant financial or non-financial interests to disclose. 


\section{REFERENCES}

[1] Orosco, R. K., Tapia, V. J., Califano, J. A., Clary, B., Cohen, E. E. W., Kane, C., Lippman, S. M., Messer, K., Molinolo, A., Murphy, J. D., Pang, J., Sacco, A., Tringale, K. R., Wallace, A. and Nguyen, Q. T., "Positive Surgical Margins in the 10 Most Common Solid Cancers," Sci. Rep. 8, 5686 (2018).

[2] Baltussen, A. J. M., Snaebjornsson, P., Brouwer de Koning, S. G., Sterenborg, H. J. C. M., Aalbers, A. G. J., Kok, N., Beets, G. L., Hendriks, B. H. W., Kuhlmann, K. F. D. and Ruers, T. J. M., "Diffuse reflectance spectroscopy as a tool for real-time tissue assessment during colorectal cancer surgery," J. Biomed. Opt. 22(10), 1-6 (2017).

[3] de Boer, L. L., Molenkamp, B. G., Bydlon, T. M., Hendriks, B. H. W., Wesseling, J., Sterenborg, H. J. C. M. and Ruers, T. J. M., "Fat/water ratios measured with diffuse reflectance spectroscopy to detect breast tumor boundaries," Breast Cancer Res. Treat. 152(3), 509-518 (2015).

[4] Brouwer De Koning, S. G., Baltussen, E. J. M., Karakullukcu, M. B., Dashtbozorg, B., Smit, L. A., Dirven, R., Hendriks, B. H. W., Sterenborg, H. J. C. M. and Ruers, T. J. M., "Toward complete oral cavity cancer resection using a handheld diffuse reflectance spectroscopy probe,” J. Biomed. Opt. 23(12), 121611 (2018).

[5] Spliethoff, J. W., Prevoo, W., Meier, M. A. J., de Jong, J., Klomp, H. M., Evers, D. J., Sterenborg, H. J. C. M., Lucassen, G. W., Hendriks, B. H. W. and Ruers, T. J. M., "Real-time in vivo tissue characterization with diffuse reflectance spectroscopy during transthoracic lung biopsy: A clinical feasibility study," Clin. Cancer Res. 22(2), 357-365 (2016).

[6] Nachabé, R., Hendriks, B. H. W., van der Voort, M., Desjardins, A. E. and Sterenborg, H. J. C. M., "Estimation of biological chromophores using diffuse optical spectroscopy: benefit of extending the UV-VIS wavelength range to include 1000 to $1600 \mathrm{~nm}$," Biomed. Opt. Express 1(5), 1432-1442 (2010).

[7] Denstedt, M., Bjorgan, A., Milanič, M. and Randeberg, L. L., "Wavelet based feature extraction and visualization in hyperspectral tissue characterization," Biomed. Opt. Express 5(12), 4260-4280 (2014).

[8] Chiu, S. J., Li, X. T., Nicholas, P., Toth, C. A., Izatt, J. A. and Farsiu, S., "Automatic segmentation of seven retinal layers in SDOCT images congruent with expert manual segmentation," Opt. Express 18(18), 19413-19428 (2010)

[9] Dijkstra, E. W., “A note on two problems in connexion with graphs," Numer. Math. 1, 269-271 (1959).

[10] Smola A. J. and Schölkopf, B., “A tutorial on support vector regression,” Stat. Comput. 14(3), 199-222 (2004). 